\title{
FPGA Based Characterisation of Thin Film For Guided Mode Resonance Devices Utilizing Layer- By-Layer Deposition of Nano-Particles
}

\author{
Saurav Bhagabati ${ }^{1}$, Rajkumar J. Borah ${ }^{2}$, and ${ }^{*}$ Shekhar S. Borah ${ }^{3}$, Student Member, IEEE \\ ${ }^{3}$ borahsumanshekhar90@gmail.com
}

\begin{abstract}
Optical sensors are extensively used in different fields of engineering. Multilayer thin film manufactured utilizing the Layer-by-Layer self-assembly method has obtained many applications such as mass sensors, smoke sensors, ammonia gas sensors, membranes. At the time of synthesis, in-situ monitoring of film growth, as well as precise control of film thickness, are of excellent significance for various applications. The deposition rate and film density are two of the various parameters that determine the nature of the accumulated film. On-line monitoring of thin-film thickness has been enhanced as an inherent part for thin-film formation with wanted features. This works aims to develop a convenient simulation method to determine the thickness of the film based upon optical signal processing using FPGA and Verilog HDL.
\end{abstract}

Keywords—FPGA, Fabry-Perot interferometer, thin-film.

\section{INTRODUCTION}

The fiber-based sensing systems have some relative advantages due to reduced volume, lightweight, resistance to Electromagnetic Interference (EMI), passive structure, hightemperature execution, wide bandwidth, high sensitivity, environmental ruggedness, and the sense of dispersed sensing. There are many ways to control the deposition rate of a thin film such as resistance monitors, capacitance monitors, ionization monitors, and quartz crystal monitors. But, most of these techniques are restricted to particular thinfilm removal techniques. Procedures relevant to opticalmeasurements are the frequent option for in-situ sensors because they are non-invasive, can be stored outside the deposition box, and are usually indifferent to the stray charged and magnetic fields related to thin-film-fabrication devices. Further, various industrial thin-film deposition techniques utilize high-pressure, chemically reactive environments. So, optical methods are the only achievable choice for in situ measurements. Optical characterization systems influence remote applications, a decrease in system complexity, and cost-efficiency. The extensive usage of optical fiber sensors, low price, and environment-friendly nature address them as a solid competitor for controlling the maturity of thin-film in-situ [1-5].

\section{THEORITICAL BACKGROUND}

\section{A. Fabry-Perot Interferometer}

Fig. 1 shows the reflection and transmission of FabryPerot interferometric system. The changing transmission function of an etalon is produced by the opposition among the many reflections of light between the two reflecting facades. Whether the multiply returned beams are in phase or not depends on the wavelength $(\lambda)$ of the light, the angle the light moves through the etalon $(\theta)$, the thickness of the etalon (d), and the refractive index of the material between the reflecting surfaces (n). If both surfaces have a reflectance $R$, the transmittance function of the etalon [2] is given by:

$$
\mathrm{T}_{\mathrm{e}}=\frac{(1-\mathrm{R})^{2}}{1+\mathrm{R}^{2}-2 \mathrm{R} \cos \delta}
$$

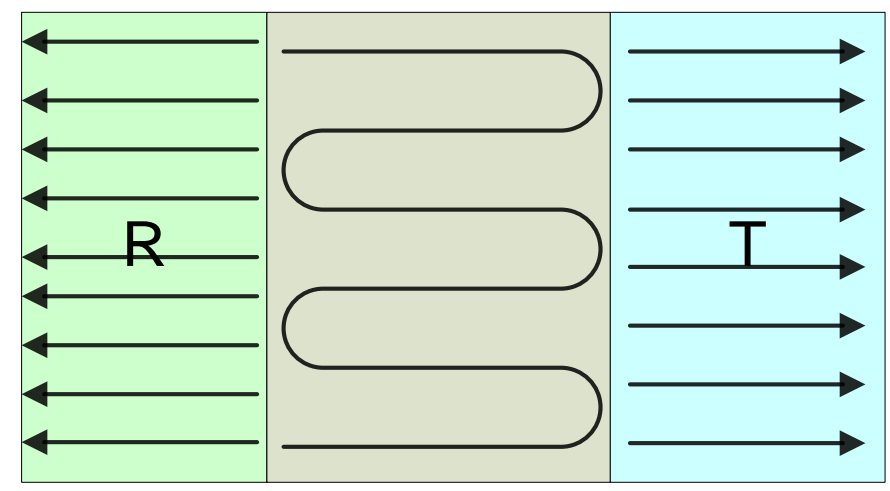

Fig. 1. Reflection and Transmission of Fabry-Perot Interferometric system

\section{B. Objectives \& Significance}

Due to the presence of the Fabry-Perot interferometer, we can accomplish repeated reflection from the multilayer thin film and through which we get constructive and destructive interference. This interference pattern can give the information related to the thickness of the thin film, for which we have to do the FFT of that interference spectrum. Using the FFT of that interference, we can say that what is the frequency component present at that spectrum. We can simulate the sensing Fabry-Perot interferometric signal for thickness range $0.5 \mu \mathrm{m}$ to $5 \mu \mathrm{m}$ with wavelength varying from $400 \mathrm{~nm}$ to $700 \mathrm{~nm}$. For a particular thickness, at first, we generate the signal and then we find the FFT of that signal. Repeating the process for the entire range of thickness. After this, we can simplify one relation between the thickness and frequency component. Using those values, we can generate a look-up table, which we can implement with FPGA, through which we can define the thickness of the thin film and can display it in the FPGA development board.to the presence of Fabry-Perot interferometer, we can accomplish repeated reflection from the multilayer thin film and through which we get constructive and destructive interference. This interference pattern can give the information related to the thickness of the thin film, for which we have to do the FFT of that interference spectrum. Using FFT of that interference, we can say that what are the frequency component present at that spectrum. 


\section{RESULTS \& DISCUSSIONS}

\section{A. Generating the Fabry-Perot Interferometric Signal}

By taking the value of the interferometer for the simulation purpose as thickness $(\mathrm{d})=1 \mu \mathrm{m}$, Refractive index, $\mathrm{n}_{1}=1.33$, refractive index of the thin film, $\mathrm{n}_{2}=1.5$, incident angle $(\theta)=0$ and, found the value of transmission for $1 \mu \mathrm{m}$ thickness from equation 1 . The simulated signal in Mod3elSim simulator is shown in Fig. 2.

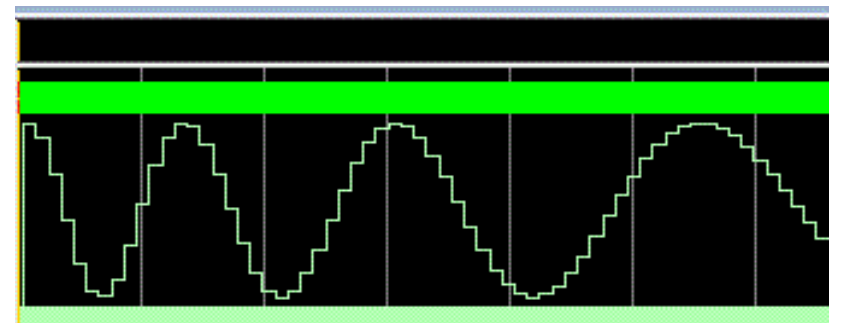

\section{1.}

Fig. 2. Simulated Signal in ModelSim Simulator

\section{B. FFT of the Interferometric Signal}

Fast Fourier Transform (FFT) gives the frequency domain representation of the Fabry Perot Interferometric sensing signal. Through which we can determine the frequency content of that spectrum. As the thickness of the film varies, it affects the phase difference of the interference pattern, which in turn affects the Fabry Perot transmission. In this way, we can calibrate the optical thickness (n.d) and frequency content $(v)$ from the FFT. The FFT is determined by this formula, where $\mathrm{x}(\mathrm{n})$ is the time-domain representation of the signal and $\mathrm{X}(\mathrm{m})$ is the frequency domain representation of that signal.

$$
X(k)=\sum_{n=0}^{N} x(n) e^{-j 2 \pi k \frac{n}{N}}
$$

For $\mathrm{k}=0,1,2 \ldots \ldots \ldots, \mathrm{N}-1$

By using FFT algorithm we can determine the frequency response of the Fabry Perot Interferometric signal for the thickness of the thin film $1 \mu \mathrm{m}$. If we vary the thickness from $0.5 \mu \mathrm{m}$ to $5 \mu \mathrm{m}$, we can get different frequency responses for respective thickness. Fig. 3 displays the FFT of the FabryPerot interferometric signal for thickness $1 \mu \mathrm{m}$. In the Fig. 4 FFT spectrum of the Fabry-Perot Spectrum with various peak for different thickness of the thin film.

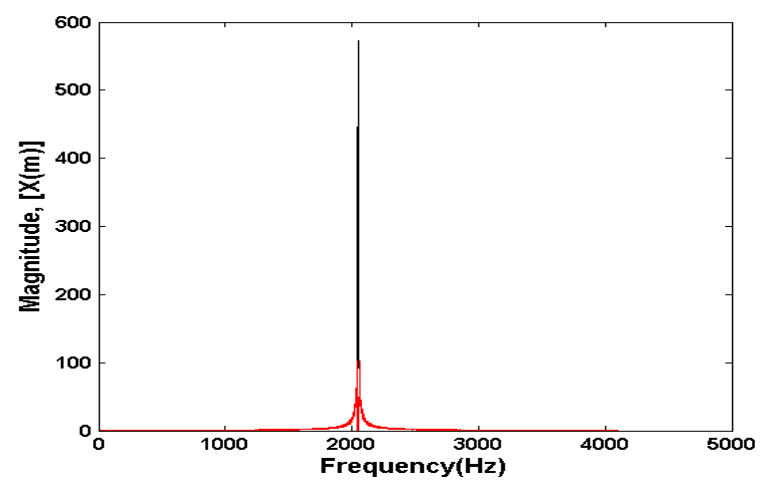

Fig. 3. FFT of the fabry perot interferometric signal For thickness $1 \mu \mathrm{m}$

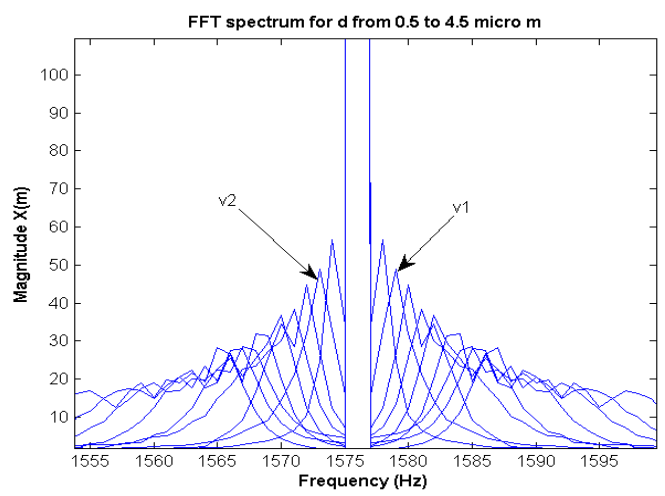

Fig. 4. FFT of the fabry perot interferometric signal For thickness range from $0.5 \mu \mathrm{m}$ to $5 \mu \mathrm{m}$

\section{Peak Detection}

For proper information of the frequency we have to remove the dc component present at that FFT spectrum. In the Fig.5, we can see the frequency peak $v_{1}$ and $v_{2}$ for respective thickness $1 \mu \mathrm{m}$. We can calculate required frequency from the two peak from this equation:

$$
\mathbf{v}=\frac{\mathbf{v}_{1}-\mathbf{v}_{2}}{2}
$$

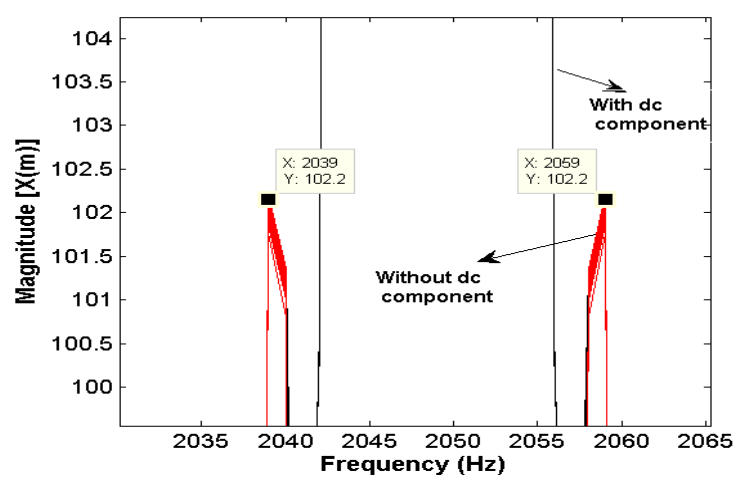

Fig. 5. Peak detection of the FFT spectrum

\section{Relation between Frequency and optical thickness}

Now we have the value of frequency component for corresponding thickness of the film. Through which we can establish a relationship between optical thickness (nd) and frequency $(v)$. Here, $n$ is the refractive index of the thin film, we assuming it 1.5 for this simulation based digital design. By plotting the values of frequency ( $v$ ) along $X$-axis and optical thickness (nd) along Y-axis, we get a graph as fig.6 given below.

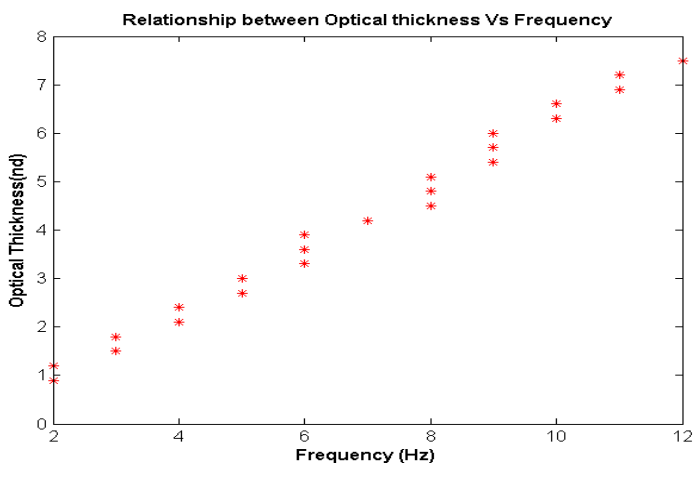

Fig. 6. Relationship curve between optical thickness and frequency. 
If we take the linear polynomial of the given parameters to establish a mathematical relation between optical thickness and frequency, then we get the relation as:

$$
\text { nd }=a_{0}+a_{1} v
$$

Where, $\mathrm{n}=$ refractive index of the thin film, $\mathrm{d}=$ thickness of the film, $\mathrm{a}_{0} \& \mathrm{a}_{1}$ proportional co-efficient, $v=$ frequency after peak detection after FFT analysis. By curve fitting analysis, we get the value of $\mathrm{a}_{0} \& \mathrm{a}_{1}$ as: $\mathrm{a}_{0}=-0.4023$ and $\mathrm{a}_{1}=$ 0.67 .

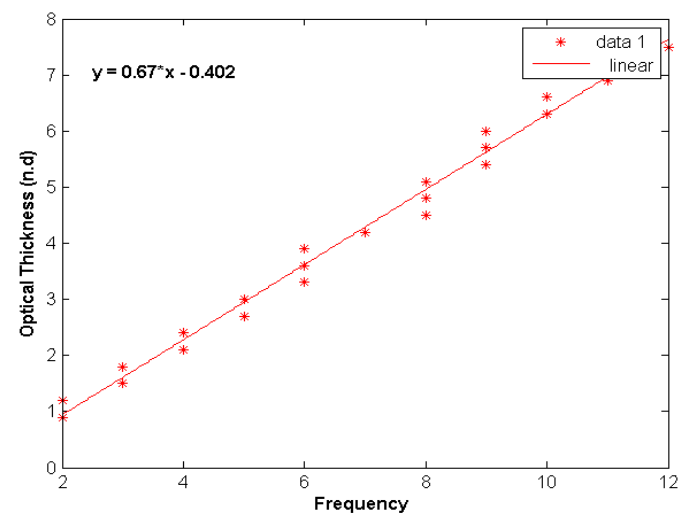

Fig. 7. Fitting curve between optical thickness and frequency

The characteristics relationship between optical thickness and frequency can defined in the LUT of digital system design, through which we can implement the Verilog coding in the FPGA development board. This work will implement in this way, if we input a Fabry -Perot Interference for unknown thickness, then the digital logic will find the FFT of the spectrum, and then it removes the dc components present at that spectrum. After removing the dc component processor can find the frequency through the digital implementation. If frequency is known to the processor, then it easily finds the thickness from the equation (4).

The digital function will implement in this way that after getting the thickness from the relationship between optical thickness and frequency, processor can display the thickness of the film in the LCD display of the FPGA development board. This work will implement with Qsys System Integration Tool \& Nios II Softprocessor.

\section{CONCLUSION}

The stated design will give a convenient, easy to use, movable, and more economical simulation system for implementation. Though SEM provides us the thickness information, using this simulation technique we can determine the thickness cost-effectively and in situ.

\section{REFERENCES}

[1] Tibuleac, S., Magnusson, R., Malonado, T. A., and Zuffada, C., 1997, "Direct and inverse techniques of guided-mode resonance filters designs," IEEE Antennas and Propagation Society International Symposium, Digest, Montreal, QC, Canada, pp. 23802383 vol.4, doi: 10.1109/APS.1997.625449.

[2] Karim, F., Bora, T., Chaudhari, M. B., Habib, K., Mohammed, W. S., Dutta, J., 2013, "Optical Fiber Based Sensor for In Situ Monitoring of Cadmium Sulfide Thin Film Growth" , vol. 38, No. 24, OPTICS LETTERS, Optical Society of America.

[3] Kotov, N. A., Dekany, I., Fendler, J. H., 1995, "Layer-by-Layer Self-Assembly of Polyelectrolyte-Semiconductor Nanoparticle Composite Films" he Journal of Physical Chemistry, 99(35), pp. 13065-13069, DOI: 10.1021/j100035a005 2012.

[4] Brown, S., Vranesic, Z., 2008, "Fundamentals of Digital logic with Verilog Design", 2nd ed., vol. 3 : McGraw-Hill.

[5] Haskell, R. E., Hanna, D. M., "Digital Design using digilent FPGA Boards", Verilog/ Active-HDL Edition, LBE Books. 